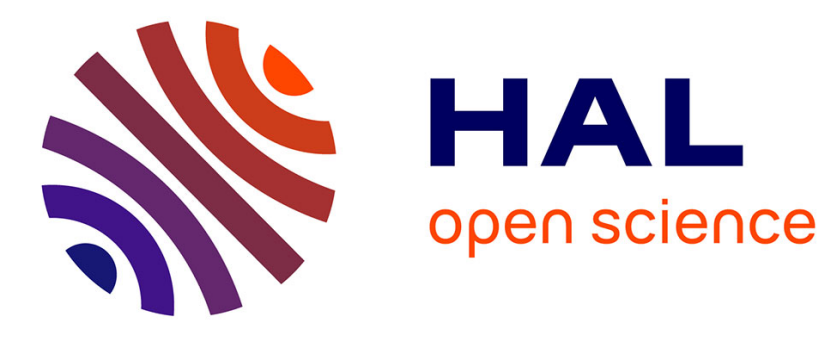

\title{
Movement Qualities as Interaction Modality
}

Sarah Fdili Alaoui, Baptiste Caramiaux, Marcos Serrano, Frédéric Bevilacqua

\section{- To cite this version:}

Sarah Fdili Alaoui, Baptiste Caramiaux, Marcos Serrano, Frédéric Bevilacqua. Movement Qualities as Interaction Modality. Designing Interactive Systems (DIS'2012), Jun 2012, Newcastle, United Kingdom. pp.1-1. hal-01161433

\section{HAL Id: hal-01161433 \\ https://hal.science/hal-01161433}

Submitted on 8 Jun 2015

HAL is a multi-disciplinary open access archive for the deposit and dissemination of scientific research documents, whether they are published or not. The documents may come from teaching and research institutions in France or abroad, or from public or private research centers.
L'archive ouverte pluridisciplinaire HAL, est destinée au dépôt et à la diffusion de documents scientifiques de niveau recherche, publiés ou non, émanant des établissements d'enseignement et de recherche français ou étrangers, des laboratoires publics ou privés. 


\section{Movement Qualities as Interaction Modality}

\author{
Sarah Fdili Alaoui \\ UPsud, LIMSI-CNRS \\ and STMS IRCAM- \\ CNRS-UPMC \\ 1 place Igor Stravinsky \\ 75004 Paris, France \\ name.surname@ircam.fr
}

\author{
Baptiste Caramiaux \\ STMS IRCAM- \\ CNRS-UPMC \\ 1 place Igor Stravinsky \\ 75004 Paris, France \\ name.surname@ircam.fr \\ Marcos Serrano \\ EnsadLab/Drii \\ 31 Rue Ulm, \\ 75005 Paris, France \\ name.surname@ensad.fr
}

\author{
Frédéric Bevilacqua \\ STMS IRCAM- \\ CNRS-UPMC \\ 1 place Igor Stravinsky \\ 75004 Paris, France \\ name.surname@ircam.fr
}

\begin{abstract}
In this paper, we explore the use of movement qualities as interaction modality. The notion of movement qualities is widely used in dance practice and can be understood as how the movement is performed, independently of its specific trajectory in space. We implemented our approach in the context of an artistic installation called $A$ light touch. This installation invites the participant to interact with a moving light spot reacting to the hand movement qualities. We conducted a user experiment that showed that such an interaction based on movement qualities tends to enhance the user experience favouring explorative and expressive usage.
\end{abstract}

\section{Author Keywords \\ Movement qualities, movement-based interaction, interaction aesthetics}

\section{ACM Classification Keywords}

H5.2. [User Interfaces]: Input devices and strategies, Interaction styles.

\section{General Terms}

Design, Experimentation.

\section{INTRODUCTION}

Human-computer interaction involving body or hand motion generally falls into two different categories: event-based or continuous motion interaction. The event-based interaction implies the detection of salient motion features, usually the start or the end of given gestures, characterized by geometrical patterns. The gestures are considered in this case as signs and symbols. Continuous motion interaction generally makes use of absolute or relative positions, forming temporal trajectories that are associated to continuous visuals (or sonic) parameters. Moreover, the correspondence between the movement and the visuals (or sonic) parameters are most often considered as direct and "instantaneous" without taking into account the movement temporal characteristics.

Permission to make digital or hard copies of all or part of this work for personal or classroom use is granted without fee provided that copies are not made or distributed for profit or commercial advantage and that copies bear this notice and the full citation on the first page. To copy otherwise, or republish, to post on servers or to redistribute to lists, requires prior specific permission and/or a fee.

DIS 2012, June 11-15, 2012, Newcastle, UK

Copyright 2012 ACM xxx-X-Xxxx-xxxx-x/xx/xx...\$10.00.
These interaction approaches, while being usually straightforward from a user perspective, take poorly into account more fundamental properties of human motion such as the notion of movement qualities. Blom et al. define movement qualities as "the distinctly observable attributes or characteristics produced by dynamics and made manifest in movement" [2]. Movement qualities result of the movement dynamics, i.e., its temporal characteristics, and can be defined independently of specific spatial trajectories, forms or shapes. This property of movement, widely used in dance practice, has barely been explored in Human-Computer Interaction (HCI).

In this paper, we propose a novel approach for HCI with movement qualities as interaction modality. We hypothesize that the use of movement qualities as modality could promote more explorative and expressive usages.

A proof of concept of this interaction modality is implemented in an artistic installation called $A$ light touch, where the participant controls a light spot through his/her hand movement qualities. The idea is to give the participant a space to express him/herself using "dance" movement qualities and provoking distinct light display behaviours. We believe that focusing on such movement characteristics actually contributes to the aesthetic aspects of the installation and enriches user experience. To evaluate this, we conducted a user experiment that investigates the body awareness and expressiveness induced by the interaction based on movement qualities.

This paper first reviews the notion of movement qualities, the existing definitions and its use in Human-Computer Interaction. Second, we describe our framework and methodology, and then we present an implementation of this approach in the context of an artistic installation. Finally, we report on the evaluation of the interaction modality and discuss the results.

\section{MOVEMENT QUALITIES REVIEW}

Movement qualities are a fundamental property of human movement that have a seminal role in dance practice. In this section we first define movement qualities as they have been formalized in both dance and psychology. Second, we present a short overview of related works in the field of HCI. 


\section{Definitions}

Among many definitions of movement qualities, we report those that can be classified as: (1) influenced by body expression and (2) influenced by motor theory.

Firstly, movement qualities as body expression were widely studied in dance-related field. Dance theorists headed by Rudolf Laban considered movement qualities as a central notion in human motion. Importantly, Laban Movement Analysis (LMA) has since been applied in various other contexts such as factory labour, early childhood development, athletics, movement therapy and other fields. For Laban, the notion of movement qualities is related to Effort, the fourth dimension of movement, the others being Body, Space and Shape dimensions [13]. For Laban, "Body" represents what is moving. "Space" is where the body is moving. "Shape" is how the body changes its shape and postures during the movement. The "Effort" dimension (formalized in a graph) describes the general characteristics about the way a movement is performed with respect to inner intention. Intention can produce changes for instance in the degree of control over the movement, the strength and the timing of the movement. Therefore, the intention affects movement dynamics and these are then intended or perceived as different movement qualities. Laban sometimes refers to the "Effort" dimension as dynamics. Hence, movement qualities and dynamics are related concepts, often confused. Blom et al. definition of movement qualities clear up the confusion: "[...] the distinctly observable attributes or characteristics produced by dynamics and made manifest in movement" [2].

Secondly, some psychologists have directly used concepts linked to movement qualities in motor theory [19]. It is defined in this field as the way human executes movements with respect to time and space. Qualities were mentioned as an important diagnostic aspect of psycho-pathological disorders in the psychiatric literature [19]. For example, the movement behaviour of schizophrenic patients is usually described as "angular, jerky, and uncoordinated, with uneven acceleration and deceleration and as either too slow or too fast a tempo" [10]. Psycho-pathological disturbances are associated to "disturbed, abrupt, halted, and stilted" gestures. Hence, terminology employed refers to kinematic and dynamic features of movements and the way they evolve from a motor control point of view. Moreover, Wallbott investigated precisely the link between emotions and movement qualities [19]. He specified three categories that showed significant differences for characterizing emotions: "movement activity" (overall quantity of motion, i.e., related to the velocity), "expansiveness/spatial extension", and "movement dynamics/energy/power". The terminology employed refers to physical systems with their dynamic, energy and power.

Formalism of movement qualities in $\mathrm{HCl}$

With the exception of [11], few HCI works have proposed body movements interactions inspired by dance.
Nevertheless some noteworthy works in movement-based interaction are inspired from LMA and particularly from Laban effort theory [13]. For example, Woo et al. proposed a vision-based $3 \mathrm{D}$ interface that extracts movement feature based on LMA [20] with the aim to bring HCI closer to human-human interaction by providing a more expressive interaction with computers. Moen [15] investigated dance theory, in particular Laban effort theory, for the design of movement-based interaction. Her first motivation was that modern and contemporary dance provides rich vocabulary for describing movement. The second motivation was its idiosyncratic nature, meaning that it encourages personal style and preferences. Finally, it is concerned with the essence of movement that is understood as the movement's qualities rather than its form.

Movement qualities are also a way to involve the user's body in experiencing the interaction with digital media. This idea is supported by Schiphorst that linked the use of movement qualities (also inspired by Laban theory) with embodied cognition and bodily experiences of aesthetics in digital arts installation [18]. Hashim et al. [9] presented a framework that provides implicit guidance for HCI research and design based on "graceful interaction". They proposed a model with Laban's movement analysis as the primary theoretical grounding of "graceful interaction" design. Finally Kjölberg suggested that giving such theory to designers makes room for body expression in interaction design [12].

These previous studies show that the use of movement qualities in HCI is promising, giving a possible framework to model the new interaction possibilities involving body expression. In this paper, we propose a general methodology for the use of movement qualities as interaction modality.

\section{FRAMEWORK AND METHODOLOGY}

Our framework proposes to focus on movement qualities, defined as how the body (or a body part) moves, independently of its specific trajectory in space. In particular, we focus on the underlying dynamics that can predict the temporal motion features. This approach is in agreement with the general concepts of movement qualities as defined in dance and psychology presented in the previous section.

The general idea thus is to build interaction techniques where the output modality (feedback/display) reacts to the user movement qualities.

We call $M Q$ interaction such interaction techniques built on movement qualities as input modality. We hypothesize that $M Q$ interaction could promote more explorative and expressive usages.

This approach can be implemented in various interaction techniques. These can vary significantly depending on the designer choices of (a) the body part and the types of movement qualities involved, (b) the modelling of the movement qualities and (c) the interaction scenarios and types of feedback and display. 
Our framework actually relies on joining together approaches and technologies (see Figure 1); it lies at the intersection of three different fields: Dance, Computer-based Recognition and Interaction Design.

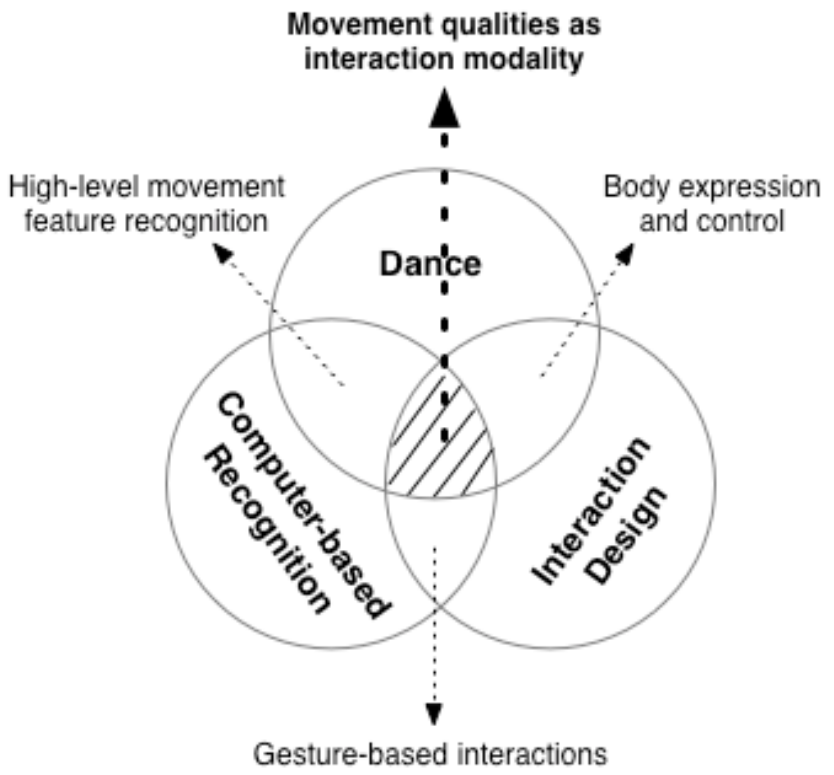

Figure 1. Framework for MQ as interaction modalities.

\section{Dance: Choice of movement qualities}

The first step is to define the interaction's input modality by building a glossary of specific labelled movement qualities. These qualities description can be provided by dance-based studies, observations or simply an idiosyncratic use in an artistic context.

\section{Computer-based recognition: Modelling and recognizing movement qualities}

According to our approach, movement qualities can be modelled by the movement's dynamics. Therefore, each quality can be characterized by the temporal evolution of the movement parameters. In some cases, it is fairly simple to find physical models that reproduce these specific dynamics. Such a modelling approach allows for real-time movement qualities recognition.

\section{Interaction design}

The third step concerns the choice of the input and output devices and the design of their relationship.

\section{PROOF OF CONCEPT}

In order to illustrate our approach, we introduce movement qualities inspired by a lasting collaboration with an internationally renowned dance company, Emio Greco | PC (EG|PC) $[6,7]$. We model EG|PC movement qualities and implement a recognition technique. Finally, we present an artistic installation where the participants can interact with a projected light spot.

\section{Choice of movement qualities}

The choice of movement qualities is motivated by dance expertise linked to choreographic research. During the last five years, we collaborated on the project "Inside Movement
Knowledge project" (IMK project) initiated by EG | PC dance company to address digital media as a potential way of documenting dance [8]. In fact, various disciplines such as linguistics, dance notations (Laban and Benesh), motion capture, digital media, recognition algorithms and glossary have been involved in the IMK project to document the company's dance vocabulary with their specific tools. Among the IMK project outputs, a glossary contains definitions, key words and qualitative descriptions of EG|PC main components, Breathing, Expanding and Reducing [8]. These components should not be understood as mere movements, but as embodying "inner intentions". These components are thus inherently linked to particular movement qualities that are bind to them.

\section{Breathing}

During the "Breathing", the body breathes in (vertical expansion until the maximum length of the body) and breathes out (releases from the maximal expansion), and again breathes in and out and so on (see Figure 2). This component has a repetitive oscillating and continuous quality.
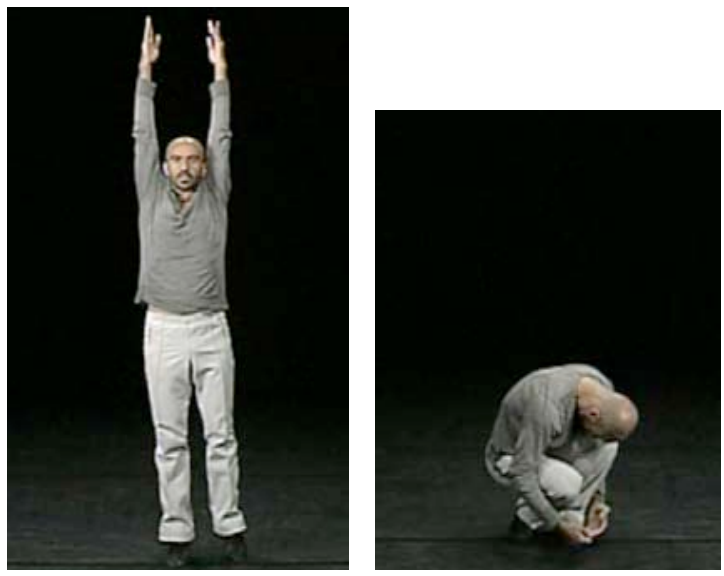

Figure 2. The "Breathing" performed by Emio Greco

\section{Expanding}

During the "Expanding", the body expands (in various directions and travels through the space) and then releases from the maximal expansion to the initial position and again expands and releases with slightly less intensity and so on until its complete cessation (see Figure 3). This component has a repetitive oscillating and viscous quality.

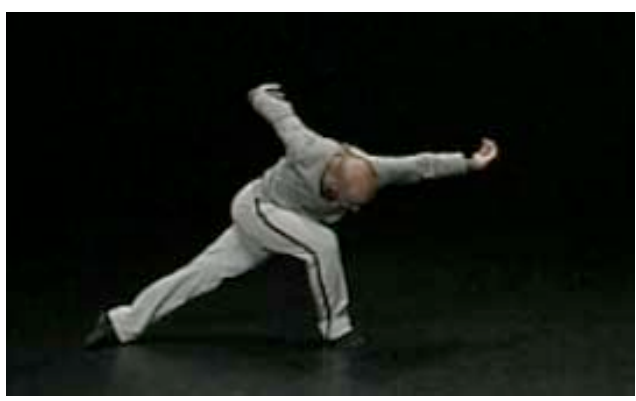

Figure 3. The "Expanding" performed by Emio Greco 


\section{Reducing}

Finally, the "Reducing" creates a specific sustained dynamic with no oscillation that slows down continuously until its complete cessation. This component has a viscous quality (see Figure 4).

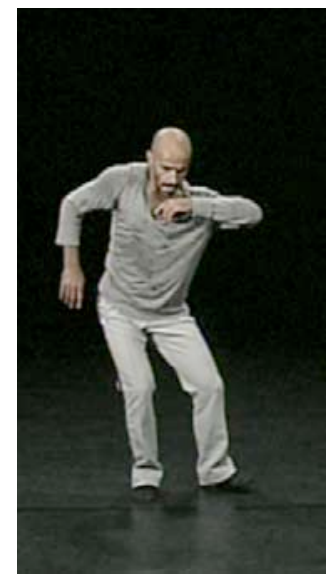

Figure 4. The "Reducing" performed by Emio Greco

\section{Modelling and recognizing movement qualities}

From a modelling point of view, movement qualities can be linked to the motion dynamics governed by a specific physical model. While this certainly represents a reduction of what movement qualities can encompass, this approach allows for the design of computational models. The goal is to formalize the considered movement dynamics through such a physical model. EG|PC movement qualities can be written as follows:

- "Breathing" has an oscillatory dynamic. The frequency of the oscillations can evolve over time;

- "Expanding" has an oscillatory dynamic that is slightly damped;

- "Reducing" has a highly damped dynamic as in a viscous environment.

Based on this formulation, these three movement qualities can be modelled as particular regimes of a second order linear differential model. They create classes linked to particular aspects of the dynamics behaviour, and not the trajectory itself. The goal is thus to fit the given physical model (given by its analytical formulation) to the motion capture incoming data by estimating the parameters that best fit these data. This is a well-known problem called system identification [14]. In the case of homogeneous linear equations (as it is the case with the previous EG|PC movement qualities), the problem can be solved using least square regression. See Figure 5 for the global illustration of the recognition method.

First we capture the data from the given interface (in the following we use a Microsoft Kinect [22]). From the captured positions, we compute the two first derivatives: velocity and acceleration. The parameter fitting is computed in a fix-length window. The length has an important role since a too short window does not allow for the recognition of movement with low oscillation frequency. On the contrary, a too large window may include two different regimes leading to a non-relevant parameter fitting. At last, the estimated parameters (in the Figure 5, only two parameters are considered) define clusters in the parameter space. Each cluster represents a specific movement quality.

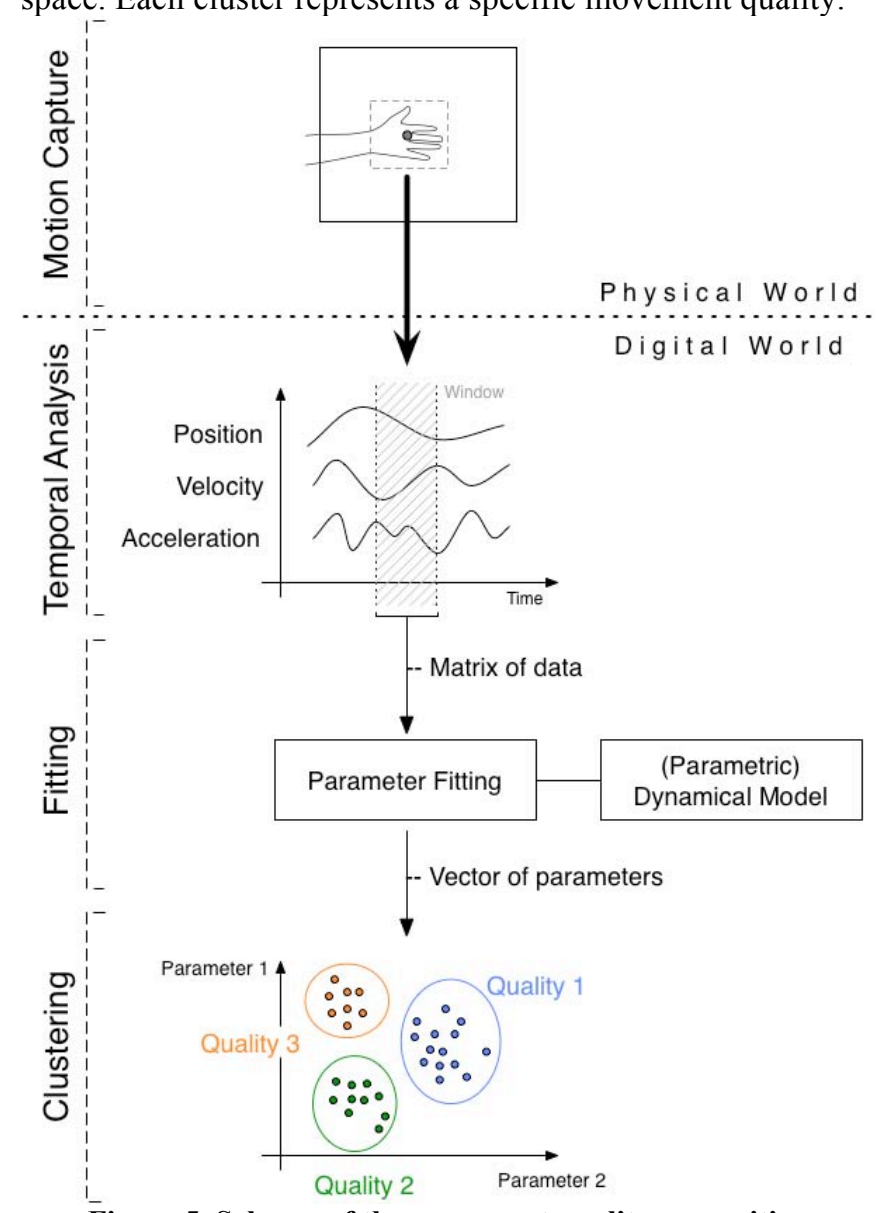

Figure 5. Schema of the movement quality recognition approach

\section{"A Light Touch" interactive installation}

We used the previously modeled movement qualities from EG|PC and the recognition process to implement an interaction technique in the context of a large-scale artistic installation called $A$ light touch.

\section{Aesthetic considerations}

Our installation allows the participant to experience the MQ interaction by interacting with their hand's movement quality inside of an empty frame and controlling a horizontal light spot projected in a rear surface (see Figure 6). The intention of this installation is to merge tangible (hand movement) and intangible (light). Here tangible is metaphorical as the name of the installation: A light touch. Inspired by light artists such as Olafur Eliasson [5], light is used here at the same time as a medium revealing the environment and as a media itself. The light spot is an evolving, living and fragile entity. Its nature is heterogeneous, sometimes close to a moving blob, 
sometimes similar to a breathing surface. Because of the subtle relationship between users movement qualities and the light, the experience of this installation is a metaphor of the movement of a hand in the water: the hand can stir the water, but the agitation of the water is partly out of its direct control (we refer the reader to the installation Liquid Views by $\mathrm{M}$. Fleischmann).

The artistic concept has been motivated by recent breakthroughs in the study of aesthetics in HCI. In our installation, the light display engages the spectator in an intrigue, improvisation and play, the three aspects defined by Peterson [17] as being part of the pragmatic aesthetics of interaction. A light touch explores the aesthetic potential of "tactility" as a pragmatic aesthetic art piece. People are invited to touch or interact and experience it [16]. A light touch focuses on the relationship between the participant and the interactive artifact, i.e., the light display [21]. It concerns the emotional threads of experience [21] rather than an aesthetic of appearance (represented by analytic aesthetic) [17].

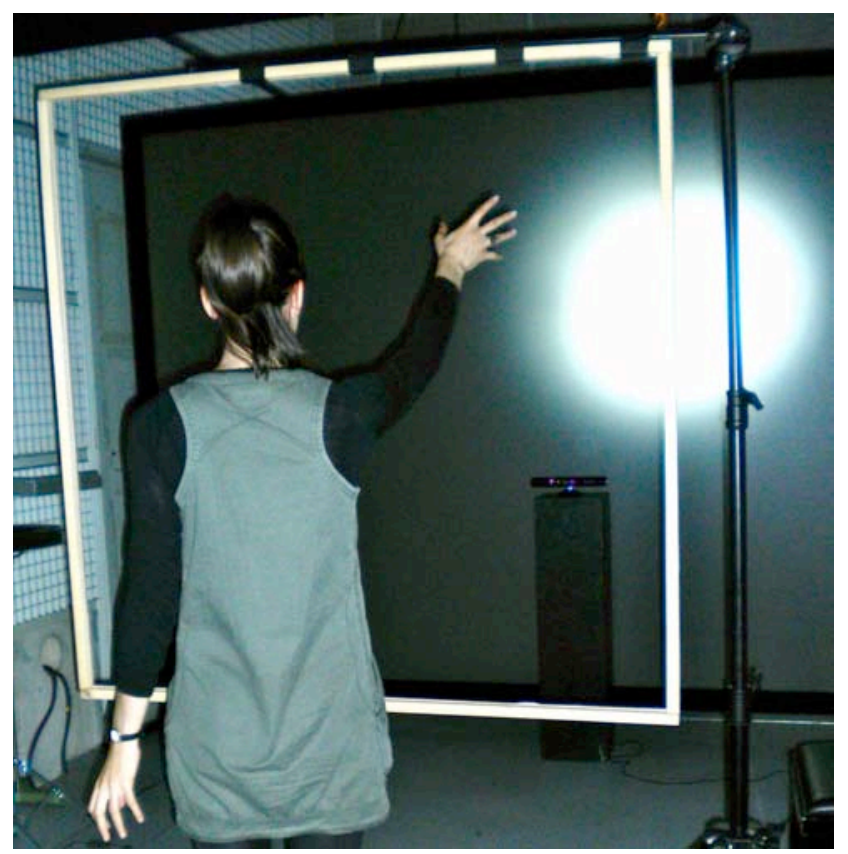

Figure 6. Photo of the installation A light touch.

\section{Scenarios}

The interaction scenarios are built as follows: The participants' movement qualities (embodied in the breathing, expanding or reducing components) are in an indirect relationship with three different light behaviours. Technically, the movement parameters provided by the movement qualities recognition are mapped to the light display as follows:

- The "Breathing" corresponds to a light blinking: movement oscillation frequency corresponds to the blinking frequency and movement energy (i.e. the norm of the motion speed) is mapped to the light intensity.
- The "Expanding" corresponds to a left-right oscillatory movement of the light spot converging to the centre as a mass attached by two springs at each side: the hand's frequency is mapped to the light frequency and the estimated dissipative effect is mapped to the speed of convergence to the centre. The hand's energy is mapped to the amplitude.

- The "Reducing" corresponds to a straight movement of the light display from left to right or right to left: the estimated dissipative effect is mapped to the speed of light evanescence and the hand's energy is mapped to the light intensity.

\section{EVALUATION}

In order to evaluate how users experience the MQ interaction in $A$ light Touch, we invited participants to explore the installation.

We aimed at comparing the MQ interaction with a standard interaction based on a direct control of the light position, called the position-based interaction ( $\mathrm{PB}$ interaction). This $\mathrm{PB}$ interaction corresponds to a one-to-one relationship where the hand's horizontal position is mapped to the light spot horizontal position and the hand vertical position is mapped to the light intensity.

\section{Participants}

We recruited 21 participants (7 female, 14 male, ages 24-49) for this study. We asked them to give us an insight on their familiarity with new technologies and in particular with interactive technologies. On average, they were all quite familiar with computers and smart phones, moderately familiar with video games, WII, Kinect and multitouch surfaces and barely familiar with motion capture and artistic interactive installation. There was no audience during the experiment.

\section{Apparatus}

The virtual "touch" interface is made of an empty square frame used to define a plane where the movements of one hand are active (see Figure 7). A Microsoft's Kinect placed in front of the screen allows for capturing the hand in the 2D Cartesian plane defined by the frame. Kinect streams the data to an OpenFrameworks application that isolates movement in the interface area using OpenCV to extract the active hand's blob. Then it sends the blob centroid 2D coordinates streamed via the OpenSoundControl (OSC) protocol to the real time programming environment Max/MSP that stores the data. The recognition program is implemented as a $\mathrm{C}++$ library and interfaced in a Max/MSP object. A mapping is then processed between the recognized characteristics of the movement and the light parameters. Two Martin led ramps with six spots each that were placed in line composes the light display apparatus. The control commands are send via DMX to the lights. Two degrees of control are available: the position along the horizontal lines and the amplitude of each spot (considered as white spots). See Figure 7 for the complete apparatus. 


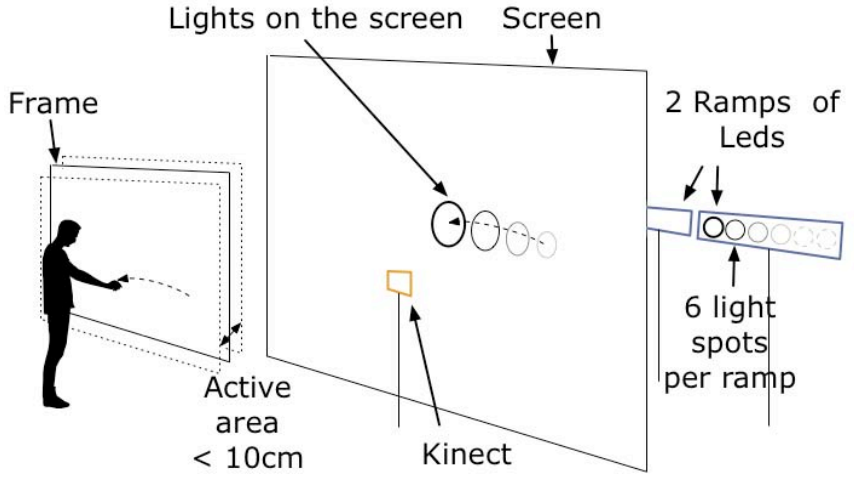

Figure 7. Installation description.

\section{Design and Procedure}

During the experiment, the participant was inside the installation space, standing up, and the investigator was sitting behind him/her. The experiment follows a withinparticipant design, meaning that all the participants went through both experimental phases and explored two interactions: $\mathrm{PB}$ and MQ interaction.

The users could control in the MQ interaction the triggering of the light behaviour (three light behaviours were available) and their frequency and damping parameters. On the other hand, the users could control in the PB interaction the light horizontal position and intensity. Note that in PB interaction, the users could have created the same light behaviours available in MQ, if they tried to simulate with their hand movement the exact wanted trajectory of the light. Thus, the same visual possibilities were achievable with the direct relationship.

The ordering of the two interaction techniques was counterbalanced across participants. For the PB interaction, we gave them an explanation of the interaction: "the light spot corresponds to the position of the hand". For the MQ interaction, we made them watch a 2 minutes video that contained explanations of the three movement qualities requested for this interaction and the corresponding light behaviours. They were asked to explore both interactions as long as they wanted. Finally, after exploring each mode, the participants were asked to answer a questionnaire. The questionnaire was presented after both explorations allowing us to compare user feedbacks for both interactions.

\section{Questionnaire}

We designed a questionnaire composed of 15 assertions according to three issues that we used for both interaction techniques to compare them in terms of the users' experience.

- First issue questions whether the interaction encourages expressivity, movement awareness and exploration and questions the richness of the interaction.

- Second issue questions whether the movements are intuitive, natural, rich, and easy to learn and perform.
- Third issue questions the relationship between movement and light behaviour.

To answer the questionnaire, users had to rate the assertions from 1 to 5 where 5 corresponded to "strongly agree" and 1 to "strongly disagree". We obtained for the questionnaire a Cronbach's alpha above 0.7 , the acceptability threshold of the American Psychological Association.

\section{RESULTS}

Our objective was to assess the user experience (UX) related to the influence of the interaction techniques: Position-based versus movement qualities. We analyzed the data with a repeated measure test on scores obtained from the questionnaire. A t-test with a significance threshold of 0.05 was computed on the scores for each answer. The results are given hereafter grouped by issues.

Overall, $71.4 \%$ of the users preferred the MQ interaction; the remaining $28,6 \%$ of the users preferred the PB interaction. The counterbalance did not affect this preference, meaning that there was no correlation between mode order and users' preference.

\section{Expressiveness, movement awareness and exploration,} richness of the interaction

\begin{tabular}{|l|l|l|c|}
\cline { 2 - 4 } \multicolumn{1}{l|}{$\begin{array}{l}\text { The exploration } \\
\text { revealed... }\end{array}$} & $\begin{array}{l}\text { PB } \\
\text { mean } \\
\text { (SD) }\end{array}$ & $\begin{array}{l}\text { MQ } \\
\text { mean } \\
(\mathrm{SD})\end{array}$ & $\begin{array}{c}\mathrm{H} 0 \text { rejection } \\
(\mathrm{P}<0.05)\end{array}$ \\
\hline $\begin{array}{l}\text { Installation } \\
\text { expressiveness? }\end{array}$ & $3.0(1.1)$ & $3.7(1.0)$ & $\begin{array}{r}\mathrm{H} 0=1, \\
\mathrm{P}=0,0375\end{array}$ \\
\hline $\begin{array}{l}\text { Light display } \\
\text { expressiveness? }\end{array}$ & $3.2(0.9)$ & $3.7(0.9)$ & $\begin{array}{r}\mathrm{H} 0=0, \\
\mathrm{P}=0,1217\end{array}$ \\
\hline $\begin{array}{l}\text { Movement } \\
\text { awareness? }\end{array}$ & $3.5(1.2)$ & $3.8(0.9)$ & $\begin{array}{r}\mathrm{H} 0=0, \\
\mathrm{P}=0,3291\end{array}$ \\
\hline $\begin{array}{l}\text { Movement } \\
\text { exploration? }\end{array}$ & $2.4(1.4)$ & $3.5(1.4)$ & $\begin{array}{r}\mathrm{H} 0=1, \\
\mathrm{P}=0,0125\end{array}$ \\
\hline $\begin{array}{l}\text { Richness of } \\
\text { interaction? }\end{array}$ & $2.4(1.1)$ & $3.5(0.8)$ & $\begin{array}{r}\mathrm{H} 0=1, \\
\mathrm{P}=0,0025\end{array}$ \\
\hline
\end{tabular}

Table 1. Findings related to expressiveness, movement awareness and exploration and richness of interaction in PB and MQ interactions.

Table 1 reports on the users' feedbacks concerning the installation and the light display expressiveness, movement awareness and exploration, and the richness of interaction. The means, standard deviations and the t-test results for each of the five assertions were computed (there were 21 scores for each interaction technique and each assertion).

\section{Expressiveness}

First, the users considered the whole installation (feedback and interaction) to be significantly more expressive in MQ than $\mathrm{PB}$ interactions $(\mathrm{H} 0=1, \mathrm{p}=0,0375)$.

No significant difference between both $\mathrm{MQ}$ and $\mathrm{PB}$ interactions were found concerning the expressiveness of the light display alone (global mean = 3.5). Although the mappings between the users' movement and the light behaviours were different in MQ than in PB interaction, we believe that the users found both light displays equally 
expressive because the intrinsic expressive nature of the light display was similar in both modes. This confirms that the perceived differences between the MQ and the PB are due to the interaction modalities and not the light display.

\section{Movement awareness}

The movement awareness induced in the installation was not significantly different between both interactions (global mean $=3.7$ ). This absence of significant differences might be related to the fact that several users were unfamiliar with the concept of the movement awareness (used in advanced dance practice).

\section{Movement exploration}

Users considered that the MQ interaction encouraged them to explore new and different movements, compared to the $\mathrm{PB}$ interaction $(\mathrm{H} 0=1, \mathrm{p}=0,0125)$. This was also supported by the fact that participants spent on average more time exploring the MQ interaction (global mean $=5 \mathrm{~min}$ ) than the $\mathrm{PB}$ interaction (global mean $=1 \mathrm{~min})$.

\section{Richness of interaction}

We investigated the assertions related to the richness of the interaction. The MQ interaction seemed to provide a richer interaction compared to the direct strategy proposed in the $\mathrm{PB}$ interaction $(\mathrm{H} 0=1, \mathrm{p}=0,0025)$. This could be linked to the previous result concerning the fostering of movement exploration by MQ interaction.

\section{Movement qualifications}

\begin{tabular}{|l|c|c|c|}
\cline { 2 - 4 } \multicolumn{1}{l|}{ Movements are ... } & PB & MQ & $\begin{array}{c}\text { H0 rejection } \\
(\mathrm{P}<0.05)\end{array}$ \\
\hline Easy to learn? & $4.5(0.6)$ & $3.3(1.1)$ & $\begin{array}{r}\mathrm{H} 0=1, \\
\mathrm{P}=0,0001\end{array}$ \\
\hline Easy to perform? & $4.7(0.5)$ & $3.5(0.9)$ & $\begin{array}{r}\mathrm{H} 0=1, \\
\mathrm{P}=0\end{array}$ \\
\hline Natural? & $3.5(1.2)$ & $2.9(1.2)$ & $\begin{array}{r}\mathrm{H} 0=0, \\
\mathrm{P}=0,1066\end{array}$ \\
\hline Intuitive? & $3.7(1.0)$ & $3.0(1.0)$ & $\begin{array}{r}\mathrm{H} 0=0, \\
\mathrm{P}=0,0731\end{array}$ \\
\hline Rich? & $2.3(1.1)$ & $3.2(1.1)$ & $\begin{array}{r}\mathrm{H} 0=1, \\
\mathrm{P}=0,0119\end{array}$ \\
\hline
\end{tabular}

Table 2. Findings related to the ease of learning and performing, the naturalness, intuitiveness and richness of movements performed in PB and MQ interactions.

In this section, the evaluation focused on motor related aspects of the movement (learning, performing) as well as its naturalness, intuitiveness and richness. Table 2 reports the means, standard deviations, and the t-test results for each of the five assertions obtained from 42 scores.

\section{Ease of learning and performing the movements}

The hand movements were found to be significantly easier to learn in the $\mathrm{PB}$ interaction than the MQ interaction $(\mathrm{H} 0=1$, $\mathrm{p}=0,0001)$. Also, the users considered that the movements are easier to perform in the PB interaction than the MQ one $(\mathrm{H} 0=1, \mathrm{p}=0)$. These two results reported in table 2 , revealed that performing movement qualities required a longer learning and training process.
Naturalness, intuitiveness and richness of the movements Movements were found fairly natural (global mean $=3.2$ ) and intuitive (global mean $=3.3$ ) with no significant difference for both interactions. However, movements were perceived richer with the MQ interaction $(\mathrm{H} 0=1, \mathrm{p}=0,0119)$.

The last two results seem to indicate that the perception of the movement easiness is counterbalanced by a loss in richness.

\section{Relationship between the user hand and the light display}

\begin{tabular}{l|c|c|c|}
\cline { 2 - 4 } $\begin{array}{l}\text { The relationship } \\
\text { between the hand and } \\
\text { the light display is }\end{array}$ & $\mathrm{PB}$ & $\mathrm{MQ}$ & $\begin{array}{c}\mathrm{H} 0 \text { rejection } \\
(\mathrm{P}<0.05)\end{array}$ \\
\hline $\begin{array}{l}\text { Not mimicry of the } \\
\text { hand trajectory? }\end{array}$ & $2.1(1.1)$ & $3.7(1.1)$ & $\begin{array}{r}\mathrm{H} 0=1, \\
\mathrm{P}=0\end{array}$ \\
\hline $\begin{array}{l}\text { A dialogue between } \\
\text { movement qualities and } \\
\text { the light behaviors? }\end{array}$ & $3.2(1.0)$ & $3.9(0.9)$ & $\begin{array}{r}\mathrm{H} 0=1, \\
\mathrm{P}=0,0405\end{array}$ \\
\hline Interesting & $3.8(0.9)$ & $4.2(0.8)$ & $\begin{array}{r}\mathrm{H} 0=0, \\
\mathrm{P}=0,1333\end{array}$ \\
\hline Intuitive & $4.0(0.9)$ & $3.1(1.1)$ & $\begin{array}{r}\mathrm{H} 0=1, \\
\mathrm{P}=0,0066\end{array}$ \\
\hline Intriguing & $2.9(1.4)$ & $3.9(1.1)$ & $\begin{array}{r}\mathrm{H} 0=1, \\
\mathrm{P}=0,0178\end{array}$ \\
\hline
\end{tabular}

Table 3. Findings related to the relationship between the users' hand and the light feedback in PB and MQ interactions.

Here, we aimed at evaluating the UX concerning the relationship between the input modality (movement qualities or positions) and the output modality (light display). The evaluation criterion is the perception of the relationships either as mimicry or as a dialogue. Moreover, the users were asked to rate if they found these relationships "interesting", "intuitive" or "intriguing". Table 3 reports the means, standard deviations, and the t-test results for each of the five assertions obtained from 42 scores. We detail the results hereafter.

\section{Mimicry versus dialogue}

The results were consistent with what we expected based on the design of the two interaction techniques.

The participants found that the light display mimicked much less the trajectory of the hand in the MQ interaction than in the $\mathrm{PB}$ interaction. The scores were significantly distinct between both interactions $(\mathrm{H} 0=1, \mathrm{p}=0)$.

The participants significantly better experienced a dialogue between their hand movement qualities and the light behaviours in the MQ interaction than the $\mathrm{PB}$ interaction $(\mathrm{H} 0=1, \mathrm{p}=0,0405)$.

\section{Relationship description}

For both interactions, the participants found the relationship very "interesting" (global mean = 3.9). As expected, they found that the relationship with the light display is significantly more "intuitive" when the light spot followed the hand's position in the $\mathrm{PB}$ interaction $(\mathrm{H} 0=1, \mathrm{p}=0,0066)$. However, they found that using movement qualities provided 
a more "intriguing" relationship with the light display $(\mathrm{H} 0=1$, $\mathrm{p}=0,0178$ ).

\section{DISCUSSION}

In this paper we studied the benefits of designing movement qualities based interaction compared to interaction based on direct relations between position and visual parameters. Our results indicate that movement qualities interaction favours expressiveness, movement exploration. Movement qualities provide a richer interaction vocabulary (but seem less intuitive, harder to learn and perform). They provide an intriguing relationship between users' movement and output modality.

This was also consistent with the longer time spent by the user in the movement quality interaction. The user explored different movements while watching the light display. Note that the exact same possibilities in terms of visual feedback were achievable with the direct relationship. Thus, the longer time spent in the installation cannot be attributed to the visual display alone. Nevertheless, it can be explained by an increased interest in the range of possibilities offered by the interaction itself.

Our study also showed that the movement qualities are not as easy to learn or perform. This might be related to the fact that we are nowadays used to technologies such as tactile surfaces that make use of continuous control with simple gestures. The users in our experiment had less experience in body expression in interactive system or digital arts installation. Nevertheless, they agree on the "richness" provided by movement qualities.

The use of movement qualities in the interaction implies a response of the system that is slightly delayed, since the system must integrate the movement variations over a short time window. The system behaves as having a short-term memory. This creates a situation that can be apprehended by the user more as a "dialogue" than a "control": The system seems to guess an "intention" of the user.

Interestingly, these two interaction modalities presented in this study can be related to the distinction between two interaction paradigms reported in [1]: computer-as-tool (parameters mapping) to computer-as-partner (movement qualities).

\section{CONCLUSION AND PERSPECTIVE}

In this paper, we explored the use of movement qualities as interaction modality. We presented an interaction technique based on movement qualities and used it in an artistic installation called $A$ light touch. In this installation, participants could interact with a light spot in a free way in order to explore the relationship between their hand movement quality and the light display behaviour.

We evaluated our interaction, comparing it with a direct manipulation interaction technique. Our experiment showed a statistical preference for the interaction based on movement qualities. Our interaction technique was perceived as a richer engaging experience, favouring wider possibilities of interaction. In this sense, this modality appears richer but also more difficult to perform.

Overall, our study confirmed that the use of movement qualities could be appropriate as interaction modality for digital arts, as discussed by $[13,16]$. For example, applications for music interaction could be designed based on this approach. Actually, movement qualities can be compared to dynamical musical entities such as legato, forte, dolce, and so forth [4]. This could achieve a continuous dialogue between the instrument and the user as proposed by Chadabe [3]. He distinguished between systems that rely on mapping of parameters and interactive systems in music. The first ones are deterministic and follow the traditional musical instrument paradigm. The second ones are interactive since they provide a constant dialogue between the performer and the created feedback.

In the future, we envision going further in the algorithmic implementation of a general movement quality recognition model. We also envisage applying movement qualities to other interactive installations and systems. We plan to build new interaction technique with movement qualities modality for music interfaces, and carry on evaluations that would complement this current study.

\section{ACKNOWLEDGMENTS}

We warmly thank Professor Christian Jacquemin for his research advices and help in the manuscript. We also thank Bertha Bermudez, Chris Ziegler, Dr. Scott deLahunta and the Emio Greco | Pieter C. Scholten dance company for their collaboration in the context of the "Inside Movement Knowledge" project. Finally we thank EnsadLab and Ircam production teams for providing the installation material.

\section{REFERENCES}

1. Beaudouin-Lafon, M. Designing interaction, not interfaces. Proceedings of the working conference on Advanced visual interfaces - AVI '04, ACM Press (2004), 15.

2. Blom, L.A. and Chaplin, L.T. The intimate act of choreography. Pittsburgh, Pa. : University of Pittsburgh Press, 1982.

3. Chadabe, J. The Limitations of Mapping as a Structural Descriptive in Electronic Instruments Joel Chadabe. New Interfaces for Musical Expression (NIME 2002), (2002), 1-5.

4. Chi, D., Costa, M., Zhao, L., and Badler, N. The EMOTE model for effort and shape. Proceedings of the 27th annual conference on Computer graphics and interactive techniques - SIGGRAPH '00, ACM Press (2000), 173-182.

5. Eliasson, O. and Yansong, M. Feelings Are Facts. Exhibition catalogue. Exhibition catalogue. Beijing: Ullens Center for Contemporary Art; Shijiwenjing Co., (2010).

6. Fdili Alaoui S., Bevilacqua F., Bermudez B., Jacquemin C. Dance Interaction with physical model visuals based 
on movement qualities. International Journal of Arts and Technology, IJART, (2012), 4-21.

7. Fdili Alaoui, S., Caramiaux, B., and Serrano, M. From dance to touch: movement qualities for interaction design. Proceedings of the 2011 ACM SIGCHI, Extended Abstract, (2011).

8. Fernandes, C. and Bermúdez, B. Inventing the interactive glossary: an approach to documenting contemporary dance. Arti Journal 2, 2 (2010), 23-25.

9. Hashim, W., Noor, N., and Adnan, W. The Design of Aesthetic Interaction: Towards a Graceful Interaction Framework. Human Development, (2009), 24-26.

10. Hietanen, J.K., Leppänen, J.M., and Lehtonen, U. Perception of Emotions in the Hand Movement Quality of Finnish Sign Language. Journal of Nonverbal Behavior 28, 1 (2004), 53-64.

11. James, J., Ingalls, T., Qian, G., et al. Movement-based interactive dance performance. Proceedings of the 14th annual ACM international conference on Multimedia Multimedia '06, ACM Press (2006), 470.

12. Kjölberg, J. Designing full body movement interaction using modern dance as a starting point. Proceedings of the 2004 conference on Designing interactive systems processes, practices, methods, and techniques - DIS '04, ACM Press (2004), 353.

13. Laban, R. Modern Educational Dance. Macdonald \& Evans, London, 1963.
14. Ljung, L. System Identification: Theory for the User. Prentice-Hall, 1987.

15. Moen, J. From Hand-Held to Body-Worn : Embodied Experiences of the Design and Use of a Wearable Movement-Based Interaction Concept. TEI '07 Proceedings of the 1st international conference on Tangible and embedded interaction, (2007), 15-17.

16. Motamedi, N. 1 Introduction : Do Not Touch 2 Touch : The Neglected Sense. Art and Design, August (2007), 22-25.

17. Petersen, M.G., Iversen, O.S., and Krogh, P.G. Aesthetic Interaction - A Pragmatist' s Aesthetics of Interactive Systems. Media, 269-276.

18. Schiphorst, T. soft ( $\mathrm{n}$ ): Toward a Somaesthetics of Touch. Hand, The, (2009), 2427-2438.

19. Wallbott, H.G. Bodily expression of emotion. European Journal of Social Psychology 28, 6 (1998), 879-896.

20. Woo, W., Kim, N., Wong, K., and Tadenuma, M. Sketch on Dynamic Gesture Tracking and Analysis Exploiting Vision-based 3D Interface. Proc. SPIE PWEI-VCIP'01, vol 4310, (2000), 656-666.

21. Wright, P., Wallace, J., and McCarthy, J. Aesthetics and experience-centered design. ACM Transactions on Computer-Human Interaction 15, 4 (2008), 1-21.

22. Microsoft Kinect. http://www.microsoft.com/enus/kinectforwindows/. 